\title{
A molecular orbital investigation of chemisorption. II. Nitrogen on tungsten (100) surface
}

\author{
Leon W. Anders and Robert S. Hansen* \\ Ames Laboratory and Department of Chemistry, Lowa State University, Ames, Iowa 50010
}

L. S. Bartell

Department of Chemistry, University of Michigan, Ann Arbor, Michigan 48104

(Received 16 August 1973)

The relative bonding energies of nitrogen chemisorbed at three symmetric sites on a $W(100)$ surface, represented by finite arrays of tungsten atoms [L. W. Anders. R. S. Hansen, and L. S. Bartell, J. Chem. Phys. 59, 5277 (1973)] were obtained by means of the extended Hückel molecular orbital theory (EHMO). The preferred site for nitrogen chemisorption was found to be the five coordination number $(5 \mathrm{CN})$ site or the fourfold site with a tungsten atom below four tungsten atoms surrounding the nitrogen atom. The $5 p$ orbital repulsive energy, in the case of hydrogen chemisorption, could be adequately approximated by the sum over pairs of empirical exponential repulsive terms; in the case of nitrogen chemisorption, this same method was approximately $10 \%$ in error at the equilibrium bond distance, and repulsive energies were therefore obtained from calculations including tungsten $5 p$ orbitals but with smaller arrays.

\section{INTRODUCTION}

In a previous paper, ${ }^{1}$ the extended Hückel molecular orbital (EHMO) technique was applied to the bonding of hydrogen to a finite cluster of tungsten atoms representing a truncated tungsten (100) surface. The relative stabilities of hydrogen bonded at the three symmetric sites of a tungsten (100) surface were compared. In addition, the contributions made by the various orbitals of the surface atoms were investigated. According to the calculations, hydrogen will preferentially chemisorb on top of a surface tungsten atom forming a bond closely resembling that of a $\mathrm{WH}$ diatomic molecule.

In this paper, this same molecular orbital (EHMO) technique has been used to investigate the bonding of nitrogen on a tungsten (100) surface. The nitrogen atom differs from the hydrogen atom particularly in its ability to form multiple bonds with the surface. The present calculations should be able to assess the influence of the additional nitrogen orbitals on a preferred chemisorption site.

\section{PROCEDURE}

The EHMO theory as formulated by Hoffmann ${ }^{2}$ and described in our previous paper ${ }^{1}$ has been used to inves- tigate the preferred structure of the $\mathrm{W}_{n} \mathrm{~N}$ surface "molecule" consisting of nitrogen chemisorbed on a truncated W (100) surface. The surface arrays which represent the three symmetric sites for chemisorption on this surface are shown in Fig. 1. These are the same sites previously described. ${ }^{1}$

Table I lists the orbital exponents for the Slater type orbitals (STO) of the nitrogen $2 s$ and $2 p$ orbitals derived from Slater's rules. ${ }^{3}$ It also includes values, taken from the work of Basch, Viste, and Gray, ${ }^{4}$ for the parameters $A, B$, and $C$ which are needed to calculate the valence orbital ionization potential (VOIP) as a function of charge, ${ }^{1}$ i. e.,

$$
\text { VOIP }=A q^{2}+B q+C
$$

The EHMO technique was first applied to the WN diatomic molecule. A comparison of Curves $a$ and $b$ in Fig. 2 shows that it is necessary to include the $5 p$ orbitals of the tungsten atom as well as the valence orbitals in the basis set to obtain a characteristic potential energy curve. The population of these nonbonding orbitals provides the repulsion needed at small internuclear separations. This repulsive energy can be approximated by

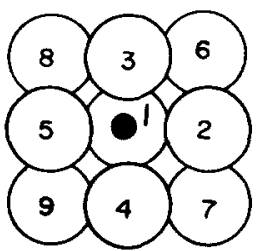

(a) $5 \mathrm{CN}$ SITE

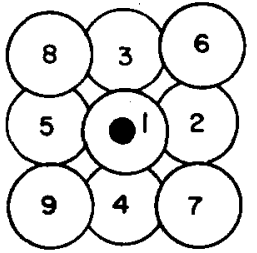

(b) ICN SITE

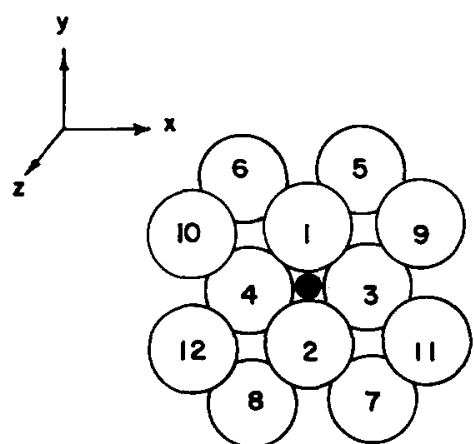

(c) 2 CN SITE
FIG. 1. Surface arrays used to model adsorption on different $W(100)$ surface sites. 
TABLE I. Orbital exponents for STO of the nitrogen atom and parameters needed to calculate VOIP as a function of atomic charge.

\begin{tabular}{|c|c|c|c|c|}
\hline Orbital & Orbital exponent & $\begin{array}{l}A \\
{\left[\mathrm{eV} /(e)^{2}\right]}\end{array}$ & $\begin{array}{l}B \\
{[\mathrm{e} V / e]}\end{array}$ & $\begin{array}{l}C \\
(\mathrm{eV})\end{array}$ \\
\hline $2 s$ & 1.950 & 3.48 & 20.25 & 28.02 \\
\hline $2 p$ & 1.950 & 3.72 & 14.14 & 16.05 \\
\hline
\end{tabular}

$$
E_{R}=A_{e}^{-r / \alpha} .
$$

As the nitrogen atom forms a surface molecule, hopefully the repulsive energy for nitrogen interacting with a larger group of tungsten atoms can be approximated by the pairwise summation of exponential terms according to $\mathrm{Eq}$. (2).

\section{RESULTS}

\section{A. Diatomic molecule and approximation of $\mathrm{W} 5 p$ orbital repulsive energy}

In Fig. 2, the bonding energy is plotted as a function of internuclear separation $r$ along the molecular axis $z$ for the WN molecule. The bonding energy was calculat ed by subtracting from the total energy (energy of the occupied molecular orbitals), the energy of the ions of the $\mathrm{W}-\mathrm{N}$ molecule at infinite separation. ${ }^{1}$ The difference between curves $a$ and $b$ in Fig. 2 is the repulsive energy resulting from the presence of the $5 p$ orbitals of tungsten in the basis set. In Fig. $3, \ln E_{\text {rop }}$ is plotted as a function of internuclear separation $r$. Values for the parameters $A$ and $\alpha$ needed to calculate the repulsive energy according to Eq. (2) were found from this plot to be $A_{W N}=2324.8 \mathrm{eV}$ and $\alpha_{W N}=0.234 \AA^{-1}$. For the final iteration, the charge on the nitrogen atom in the WN molecule at equilibrium separation was -0.38 and that on the tungsten atom +0.38 .

Single bond covalent radii $(0.70 \AA$ for $\mathrm{N}$ and $1.30 \AA$ for $W$ ) predict a length of $2.00 \AA$ for a $W-N$ single bond. Pauling's empirical relationship ${ }^{5}$ between bond length and bond order (see previous paper), ${ }^{1}$ coupled with the 1.65 A equilibrium bond length indicated by Fig. 2, gives a value of 3.1 for the bond order of the WN molecule. Table II, which lists the LCAO for the occupied
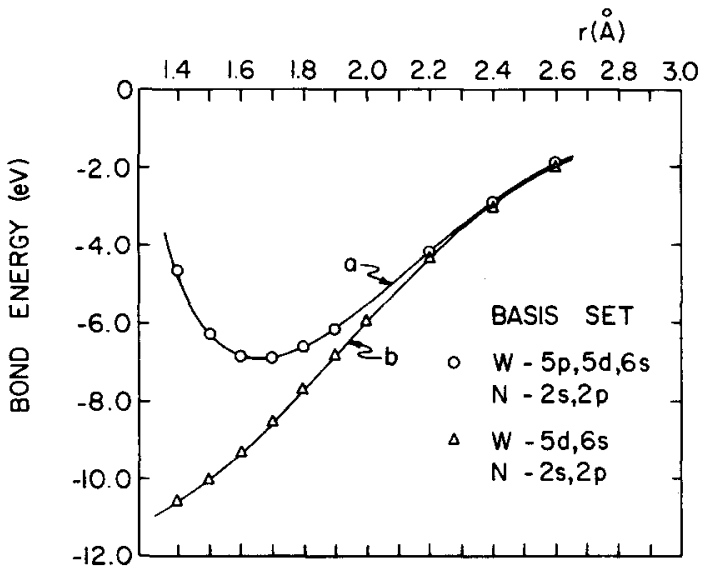

FIG. 2. Dependence of bond energy on bond distance for WN molecule with and without inclusion of $\mathrm{W} 5 p$ orbitals.

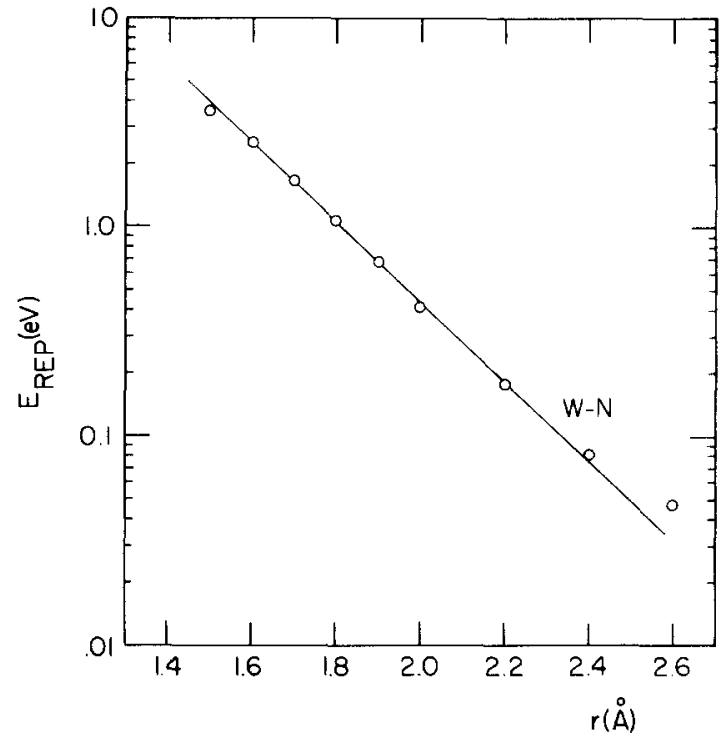

FIG. 3. Dependence of nonbonding (5p orbital) repulsive energy on distance for the WN molecules.

molecular orbitals of the WN molecule, shows that there are three molecular orbitals $\left(\Psi_{2}, \Psi_{3}\right.$, and $\left.\Psi_{4}\right)$ which make major contributions to the bonding; this suggests that the bond order of 3.1 is a plausible value.

Figure 4 compares the $\mathrm{W}_{2} \mathrm{~N}$ (The configuration used set the $\mathrm{W}-\mathrm{W}$ distance at $3.16 \AA$ and varied the $\mathrm{N}$ position along the perpendicular bisector of the $\mathrm{W}-\mathrm{W}$ bond. $r$ in Fig. 4 is $\mathrm{W}-\mathrm{N}$ distance.) molecule repulsive energies determined (curve a) as the difference between the energies calculated by the EHMO technique with the inclusion and omission of the tungsten $5 p$ orbitals and (curve b) by summing analytical exponential terms over pairwise interactions. There is about a $10 \%$ difference in the repulsive energies in the neighborhood where the chemical bond is formed. Because of this difference, larger molecules $\left(\mathrm{W}_{4} \mathrm{~N}\right.$ and $\left.\mathrm{W}_{5} \mathrm{~N}\right)$ which still lent themselves to easy computation were investigated; these molecules are reductions of the surface arrays in Fig. 1. The tungsten atoms omitted from the surface arrays in Fig. 1 for the above calculations are at a sufficient distance from the nitrogen atom at each of the symmetric

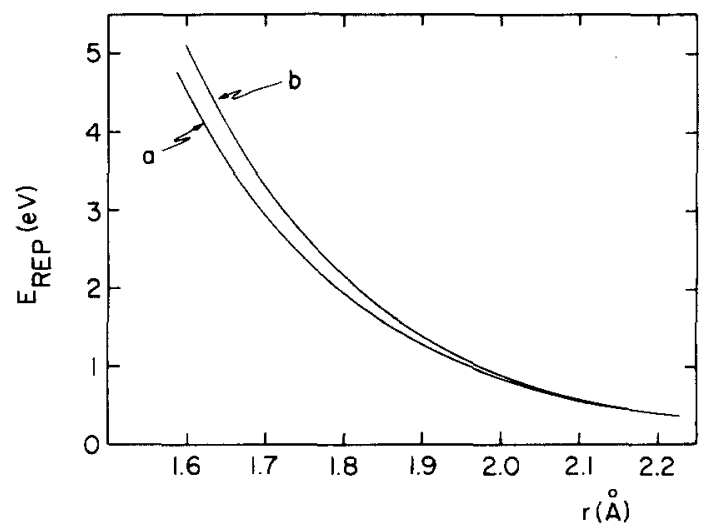

FIG. 4. Additivity of nonbonded orbital repulsive energy. (a) Difference in $E(r)$ for $\mathrm{W}_{2} \mathrm{~N}$ between calculations including and omitting W $5 p$ orbitals; (b) $E(r)$ calculated by pairwise addition of analytic repulsive energy functions. 
TABLE II. Occupied molecular orbitals for the diatomic molecule WN.

\begin{tabular}{lcccccc}
\hline \hline $\mathrm{W}-\mathrm{N}$ & $\Psi_{1}$ & $\Psi_{2}$ & $\Psi_{3}$ & $\Psi_{4}$ & $\Psi_{5}$ & $\Psi_{6}$ \\
Energy (eV) & -21.61 & -12.74 & -12.74 & -11.51 & -10.29 & -10.29 \\
\hline $\mathrm{N}$ & & & & & & \\
$2 s$ & 0.86 & & & 0.13 & & \\
$2 p_{z}$ & 0.08 & & & 0.72 & & \\
$2 p_{x}$ & & -0.69 & & & & \\
$2 p_{y}$ & & & -0.69 & & & \\
$\mathrm{~W}$ & 0.21 & & & -0.48 & & \\
$5 d_{x^{2}}$ & & -0.54 & & & & \\
$5 d_{x^{2}}$ & & & -0.54 & & & \\
$5 d_{x^{2}-y^{2}}$ & & & & & & \\
$5 d_{y s}$ & 0.13 & & & & & \\
$5 d_{x y}$ & & & & & & \\
$6 s$ & & & & & & \\
\hline \hline
\end{tabular}

sites that their $5 p$ orbitals should not contribute to the repulsive energy when the larger surface arrays are used. In Fig. 5, the repulsive energies between the nitrogen atom at each of the bonding sites and the tungsten atoms in the above molecules are plotted.

Curves $a$ in Fig. 5 are based on repulsive energies calculated from the $W_{5} \mathrm{~N}$ or $W_{4} N$ arrays as explained above, Curves $b$ by pairwise summation of analytical exponential terms. Repulsive energies at bonding distances obtained from Curves $a$ are $10 \%$ higher for the $1 \mathrm{CN}$ site, about the same for the $2 \mathrm{CN}$ site, and about $10 \%$ lower for the $5 \mathrm{CN}$ site than those obtained from Curves $b$. Approximation of repulsive energy by pairwise summation of exponential terms [Eq. (2)] was therefore judged insufficiently reliable, and it was approximated instead from direct calculations on the abbreviated arrays $W_{4} N$ and $W_{5} N$.

\section{B. Nitrogen chemisorbed on a W (100) surface}

The adjustments of the VOIP of the edge atoms of the surface arrays in Fig. 1 to reduce charge transfer were the same as those previously adopted. ${ }^{1}$ The VOIP of the orbitals of the nitrogen atom were iterated at equilibrium separation to self-consistency for charge $q$ at each of the three symmetric sites. On the final iteration, the VOIP for the $2 s$ and $2 p$ orbitals of nitrogen were -18.93 and $-10.01 \mathrm{eV}$ at the $5 \mathrm{CN}$ site, -17.17 and $-8.93 \mathrm{eV}$ at the $2 \mathrm{CN}$ site, and -17.31 and $-9.02 \mathrm{eV}$ at the $1 \mathrm{CN}$ site. To avoid possible oversensitivity of results to VOIP parametrization, the calculations were repeated $\mu$ sing -17.77 and $-9.29 \mathrm{eV}$ for the $2 s$ and $2 p$ orbitals of nitrogen, respectively, at all the sites. As in the case of hydrogen chemisorption, ${ }^{1}$ the same ordering in site preference was obtained by both methods and constant nitrogen $2 s$ and $2 p$ VOIP's were used in further calculations. The bonding energies as a function of internuclear separation are plotted in Fig. 6. The internuclear separation was the distance of nitrogen to the nearest tungsten atom(s) in the surface atom arrays. In the case of the $5 \mathrm{CN}$ site, it was the distance of the tungsten atom in the hole; for the $2 \mathrm{CN}$ site, it was the distance to the two surface atoms; and for the $1 \mathrm{CN}$ site, it was the distance to the single tungsten atom. The bonding energy $E$ was calculated according to the following relationship:

$$
E=\sum_{W_{n} \mathbb{N}} n_{i} \epsilon_{i}-\sum_{W_{n}} n_{i} \epsilon_{i}-\sum_{\mathbb{N}} n_{i} \epsilon_{i}+E_{\text {red }},
$$

where the first term in the summation over occupied molecular orbitals of energy $\epsilon_{i}$ of the $W_{n} N$ surface molecule, the next term is the summation over the occupied molecular orbitals of energies $\epsilon_{i}$ of the $\mathrm{W}_{n}$ part of the molecule at infinite separation from the nitrogen atom, and the next term is the summation over the occupied atomic orbitals of energy $\epsilon_{i}$ of the nitrogen atom, also at infinite separation. The last term is the repulsive energy $E_{\text {rep }}$ determined from the $\mathrm{W}_{5} \mathrm{~N}$ and $\mathrm{W}_{4} \mathrm{~N}$

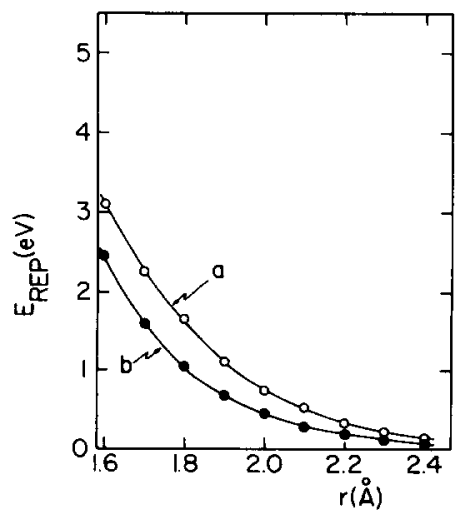

(a) $W_{5} N-1 C N$ SITE

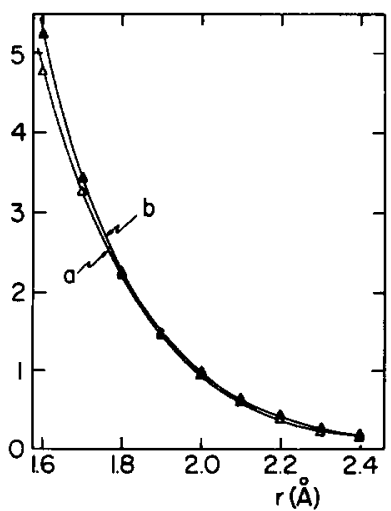

(b) $W_{4} N-2 C N$ SITE

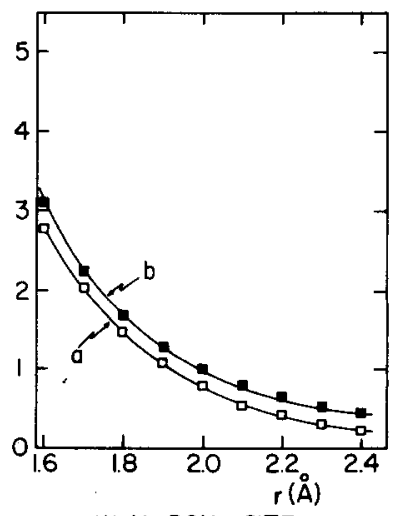

(c) $W_{5} N-5 C N$ SITE
FIG. 5. Additivity of nonbonded orbital repulsive energy of abbreviated surface arrays. (a) Difference in $E(r)$ for $\mathrm{W}_{n} \mathrm{~N}(n=4,5)$ between calculations including and omitting $\mathrm{W} 5 p$ orbitals; (b) $E(r)$ calculated by pairwise addition of analytic repulsive energy functions. 
TABLE II. Energies, equilibrium distances, and charges for nitrogen bonded to different W (100) surface sites.

\begin{tabular}{|c|c|c|c|c|}
\hline Site & $\begin{array}{l}\text { Bond energy } \\
(\breve{A})\end{array}$ & $\begin{array}{l}r_{e} \\
(\tilde{A})\end{array}$ & Charge & $\begin{array}{l}\text { Nitrogen bond } \\
\text { overlap population }\end{array}$ \\
\hline $5 \mathrm{CN}$ & -3.25 & 2.05 & -0.04 & 1.12 \\
\hline $2 \mathrm{CN}$ & -3.15 & 2.13 & -1.36 & 0.92 \\
\hline $1 \mathrm{CN}$ & -2.85 & 1.88 & -1.13 & 0.77 \\
\hline
\end{tabular}

molecules and plotted as curves a in Fig. 5. Table III summarizes the results of calculations plotted in Fig. 6 .

The surface arrays in Figs. 1(a) and 1(b) belong to the $C_{4 v}$ symmetry groups and the surface array in Fig. 1(c) to the $C_{2 v}$ symmetry group. ${ }^{1}$ In these groups, the $2 s$ and $2 p_{2}$ orbitals of nitrogen belong to the totally symmetric $a_{1}$ irreducible representation. Therefore, these orbitals of nitrogen can interact only with the totally symmetric molecular orbitals of the $\mathrm{W}_{n}$ surface arrays. In the $C_{4 v}$ symmetry group, the $2 p_{x}$ and $2 p_{y}$ orbitals of nitrogen belong to the $e$ irreducible representation and will interact with molecular orbitals of the $W_{9}$ arrays which also belong to this representation. These same orbitals of nitrogen belong to the $b_{1}\left(2 p_{x}\right)$ and $b_{2}\left(2 p_{y}\right)$ irreducible representations in the $C_{2 v}$ symmetry group and interact with the molecular orbitals of the $\mathrm{W}_{12}$ array belonging to these same irreducible representations.

Molecular orbital energy diagrams for the molecular orbitals belonging to the totally symmetric $a_{1}$ irreducible representation are shown in Fig. 7. On the left side of each panel in these figures are the molecular orbitals from the $\mathrm{W}_{n}$ arrays, on the right side are the atomic orbitals of nitrogen and in the middle are the molecular orbitals for the $\mathrm{W}_{n} \mathrm{~N}$ surface molecules. The occupied totally symmetric $\left(a_{1}\right)$ molecular orbitals in Fig. 7 are tabulated in Tables IV and $V$ for the $W_{9}$ and $W_{12}$ arrays, respectively. These can be compared with the totally symmetric molecular orbitals of the $\mathrm{W}_{n} \mathrm{~N}$ surface molecules (Table VI, $1 \mathrm{CN}$; Table VII, $2 \mathrm{CN}$; and Table VIII, $5 \mathrm{CN}$ ). The molecular orbitals of $\mathrm{W}_{n} \mathrm{~N}$ most closely resembling those of $\mathrm{W}_{n}$ surface arrays are given
$r(\AA)$

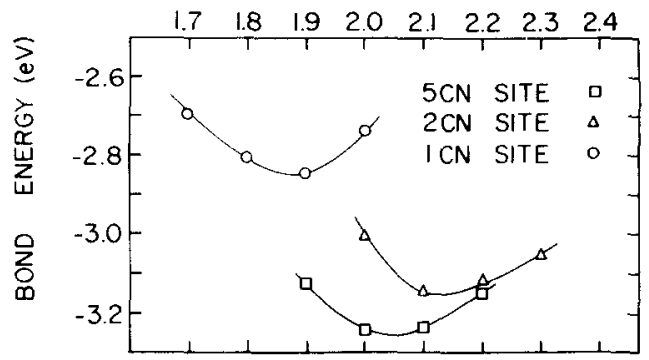

FIG. 6. Bond energies as functions of distances for nitrogen adsorbed on different $\mathrm{W}(100)$ surface sites.

corresponding subscripts and primed.

For $k$ totally symmetric orbitals in the $\mathrm{W}_{n}$ surface arrays, there should be $k+2$ totally symmetric orbitals in the $\mathrm{W}_{n} \mathrm{~N}$ surface molecule, resulting from the presence of the $2 s$ and $2 p_{2}$ totally symmetric atomic orbitals of nitrogen in the $\mathrm{W}_{n} \mathrm{~N}$ species. In the $\mathrm{W}_{9}$ arrays [Figs. $1(\mathrm{a})$ and $1(\mathrm{~b})]$, there are five occupied totally symmetric molecular orbitals. For the $\mathrm{W}_{9} \mathrm{~N}$ surface molecule with nitrogen bonded at the $1 \mathrm{CN}$ site [Fig. 7(a)], there are seven totally symmetric orbitals lower in energy than the highest occupied molecular orbital (HOMO), whereas at the $5 \mathrm{CN}$ site [Fig. 7(c)] only six are below the HOMO. At the $2 \mathrm{CN}$ site [Fig. 7(b)], there are 11 occupied totally symmetric molecular orbitals in the $\mathrm{W}_{12}$ surface arrays, and in the $\mathrm{W}_{12} \mathrm{~N}$ surface molecule there are 12 below the HOMO.

In Fig. 8, molecular orbital energy diagrams are shown which involve the $2 p_{x}$ and $2 p_{y}$ orbitals of nitrogen. In the case of the $W_{9}$ arrays for the $1 \mathrm{CN}$ and $5 \mathrm{CN}$ sites [Figs. $8(\mathrm{a})$ and $8(\mathrm{c})$ ] the se molecular orbitals belong to the $e$ ir reducible representations, and for the $2 \mathrm{CN}$ site [Fig. $8(\mathrm{~b})$ ] they belong to the $b_{1}$ and $b_{2}$ irreducible representations. The molecular orbitals represented in Fig. 8 for the $W_{9}$ and $W_{12}$ are tabulated in Tables IX and $X$, respectively. These can be compared with the molecular orbitals of the $\mathrm{W}_{n} \mathrm{~N}$ surface molecule (Table XI, $1 \mathrm{CN}$; Table XII, $2 \mathrm{CN}$; Table XII, $5 \mathrm{CN}$ ). Again

TABLE IV. Totally symmetric molecular orbitals for the surface arrays representing the $1 \mathrm{CN}$ and $5 \mathrm{CN}$ sites.

\begin{tabular}{|c|c|c|c|c|c|}
\hline $\begin{array}{l}\text { Molecular orbital } \\
\text { Energy (ev) }\end{array}$ & $\begin{array}{l}\Psi_{1} \\
(-12.43)\end{array}$ & $\begin{array}{l}\boldsymbol{\Psi}_{2} \\
(-10.69)\end{array}$ & $\begin{array}{l}\Psi_{3} \\
(-10.38)\end{array}$ & $\begin{array}{l}\Psi_{4} \\
(-9.77)\end{array}$ & $\begin{array}{l}\Psi_{5} \\
(-8.47)\end{array}$ \\
\hline \multicolumn{6}{|l|}{$W(1)$} \\
\hline $\begin{array}{l}5 d_{z^{2}} \\
6 s\end{array}$ & -0.24 & -0.54 & & -0.10 & -0.19 \\
\hline \multicolumn{6}{|l|}{$W(2,3,4,5)$} \\
\hline $5 d_{z^{2}}$ & & 0.22 & 0.17 & 0.16 & \\
\hline $5 d_{x \varepsilon}(2,5)$ & & \pm 0.15 & & \pm 0.31 & \pm 0.07 \\
\hline $5 d x^{2}-y^{2}$ & & \pm 0.12 & \pm 0.30 & \pm 0.19 & \pm 0.05 \\
\hline $5 d_{y z}(3,4)$ & & \pm 0.15 & & \pm 0.31 & ${ }_{ \pm} 0.07$ \\
\hline $6 s$ & -0.18 & & & & \\
\hline \multicolumn{6}{|l|}{$W(6,7,8,9)$} \\
\hline $5 d z^{2}$ & & -0.10 & -0.19 & & 0.26 \\
\hline $5 d_{x z}$ & & & \pm 0.11 & & \pm 0.28 \\
\hline $5 d$ & & & \pm 0.11 & & \pm 0.28 \\
\hline $5 d_{x y}$ & & \pm 0.12 & & \pm 0.22 & \pm 0.10 \\
\hline $6 s$ & -0.12 & & & & \\
\hline
\end{tabular}




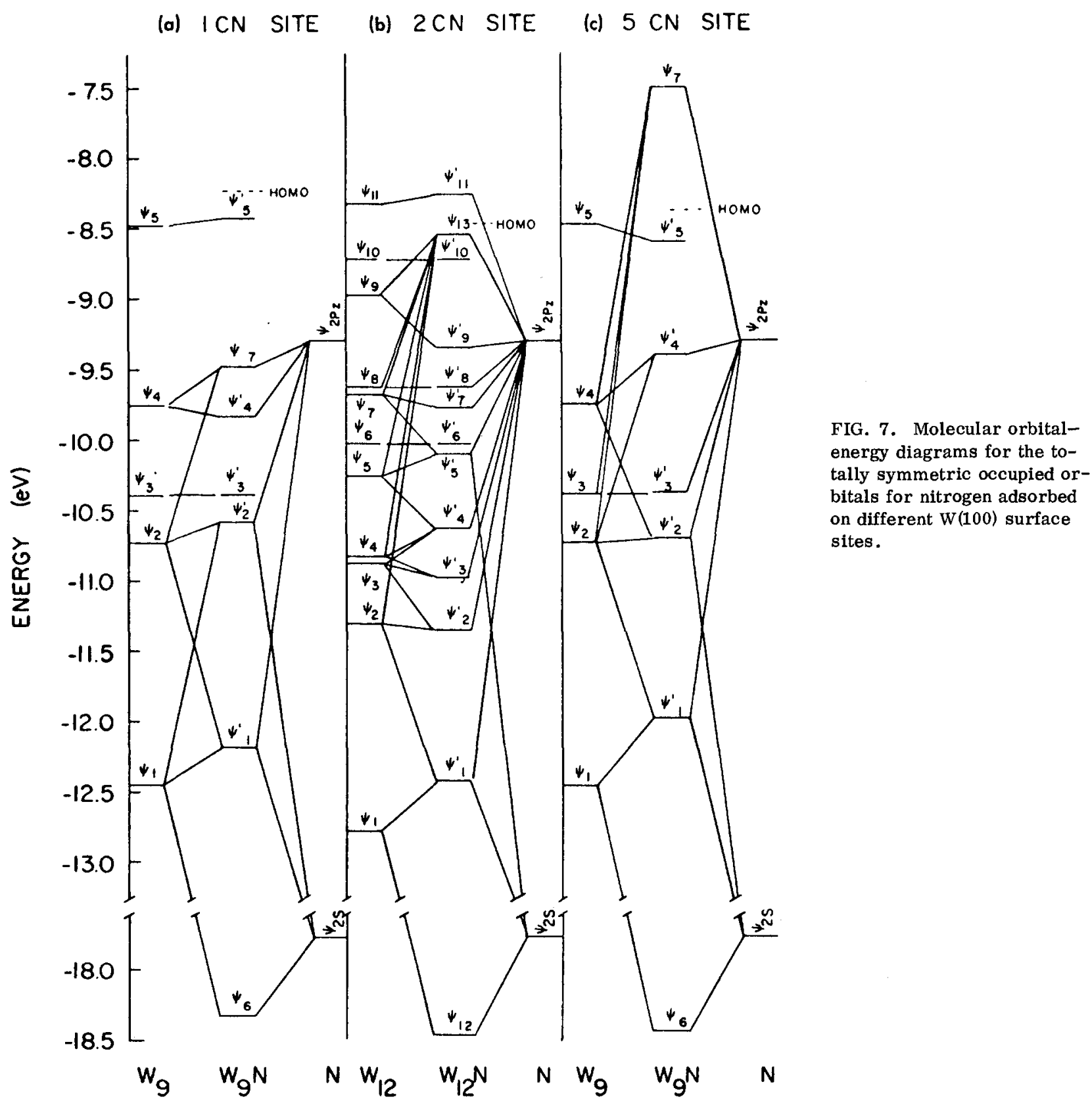

the primed molecular orbitals indicate a similarity in the contributions made by the tungsten atoms in the $W_{n}$ and $\mathrm{W}_{n} \mathrm{~N}$ cases.

There are 14 occupied molecular orbitals [Figs. 8(a) and $8(c)]$ in the $W_{9}$ array which have the same symmetry as the nitrogen $2 p_{x}$ and $2 p_{y}$ atomic orbitals, and $18 \mathrm{oc}-$ cupied molecular orbitals [Fig. $8(b)$ ] in the case of $W_{12}$ array. In the $\mathrm{W}_{n} \mathrm{~N}$ arrays, the molecular orbitals are matched with those of the $W_{n}$ arrays and are designated accordingly. The $\mathrm{W}_{0} \mathrm{~N}$ surface molecule with nitrogen at the $1 \mathrm{CN}$ and $5 \mathrm{CN}$ site have 16 molecular orbitals of $e$ symmetry which are occupied. In the case of the $\mathrm{W}_{9} \mathrm{~N}$ surface molecule with nitrogen at the $1 \mathrm{CN}$ site, molecular orbitals $\psi_{15}$ and $\psi_{16}$ are the HOMO and are singly occupied; whereas at the $5 \mathrm{CN}$ site, $\psi_{15}$ and $\psi_{16}$ are triply occupied. In the $\mathrm{W}_{12} \mathrm{~N}$ array with nitrogen bonded at the $2 \mathrm{CN}$ site, there are 20 molecular orbitals of $b_{1}$ and $b_{2}$ symmetry all below the HOMO and doubly occupied.

\section{DISCUSSION}

Moderate doses of nitrogen to a $\mathrm{W}(100)$ surface lead to a single high temperature state, desorbing by second order kinetics, according to single crystal flash desorption studies. ${ }^{6,7}$ This is most simply interpreted as reflecting dissociative adsorption of nitrogen to form nitrogen adatoms. Low energy electron diffraction (LEED) studies ${ }^{8}$ have shown that this adorption state when fully developed produces a $C(2 \times 2)$ pattern, which is most simply interpreted as due to a structure in which nitrogen adatoms are placed in alternate unit cells of the clean surface. The foregoing experiments do not further locate the nitrogen adatoms with respect to the tungsten atoms; the diffuse half-order spots in the $C(2 \times 2)$ LEED pattern $s^{8, \theta}$ favor structures in which 
TABLE V. Totally symmetric molecular orbitals for the surface array representing the $2 \mathrm{CN}$ site:

\begin{tabular}{|c|c|c|c|c|c|c|c|c|c|c|c|}
\hline $\begin{array}{l}\text { Molecular orbital } \\
\text { Energy (eV) }\end{array}$ & $\begin{array}{l}\psi_{1} \\
(-12.78)\end{array}$ & $\begin{array}{l}\psi_{2} \\
(-11.31)\end{array}$ & $\begin{array}{l}\psi_{3} \\
(-10.87)\end{array}$ & $\begin{array}{l}\psi_{4} \\
(-10.82) \\
\end{array}$ & $\begin{array}{l}\psi_{5} \\
(-10.25) \\
\end{array}$ & $\begin{array}{l}\Psi_{6} \\
(-10.03) \\
\end{array}$ & $\begin{array}{l}\psi_{7} \\
(-9.68) \\
\end{array}$ & $\begin{array}{l}\psi_{8} \\
(-9.62) \\
\end{array}$ & $\begin{array}{l}\psi_{9} \\
(-8.97) \\
\end{array}$ & $\begin{array}{l}\psi_{10} \\
(-8.72)\end{array}$ & $\begin{array}{l}\psi_{11} \\
(-8.32)\end{array}$ \\
\hline \multicolumn{12}{|l|}{$W(1,2)$} \\
\hline $5 d_{\xi^{2}}$ & & & -0.36 & -0.09 & & -0.08 & 0.09 & -0.15 & & & 0.15 \\
\hline $5 d x^{2}-y^{2}$ & & 0.33 & & & -0.19 & -0.06 & -0.05 & -0.08 & -0.30 & -0.14 & 0.08 \\
\hline $\begin{array}{l}5 d_{y s} \\
6 s\end{array}$ & 0.18 & \pm 0.12 & \pm 0.08 & \pm 0.32 & \pm 0.21 & & \pm 0.17 & \pm 0.18 & \pm 0.10 & ${ }_{ \pm} 0.12$ & \\
\hline \multicolumn{12}{|l|}{$\mathrm{W}(3,4)$} \\
\hline $5 d_{z^{2}}$ & & & 0.36 & -0.09 & & -0.08 & -0.09 & -0.15 & & & -0.15 \\
\hline $5 d_{y z}$ & & \pm 0.12 & \pm 0.08 & \pm 0.32 & \pm 0.21 & & \pm 0.17 & ${ }_{ \pm 0} 0.18$ & \pm 0.10 & \pm 0.12 & \\
\hline $5 d_{x^{2}-y^{2}}$ & & -0.33 & & & -0.19 & 0.05 & 0.05 & +0.08 & -0.30 & 0.14 & 0.08 \\
\hline $6 s$ & 0.18 & & & & & & & & & & \\
\hline \multicolumn{12}{|l|}{$W(5,6,7,8)$} \\
\hline $5 d_{z^{2}}$ & & & 0.12 & 0.05 & -0.17 & 0.07 & 0.08 & 0.06 & 0.08 & -0.12 & 0.11 \\
\hline $5 d_{x z}$ & & & \pm 0.05 & \pm 0.07 & \pm 0.14 & \pm 0.23 & & \pm 0.09 & & & ${ }_{ \pm} 0.26$ \\
\hline $5 d_{x^{2}-y^{2}}$ & & -0.13 & 0.05 & -0.11 & 0.10 & -0.16 & 0.05 & & -0.20 & -0.05 & -0.14 \\
\hline $5 d_{y z}$ & & & & & & \pm 0.07 & \pm 0.13 & \pm 0.19 & \pm 0.14 & \pm 0.24 & \pm 0.10 \\
\hline $5 d_{x y}$ & & & & \pm 0.12 & & \pm 0.08 & \pm 0.24 & \pm 0.18 & \pm 0.09 & \pm 0.18 & ${ }_{ \pm} 0.08$ \\
\hline $6 s$ & 0.10 & & & & & & & & & & \\
\hline \multicolumn{12}{|l|}{$\mathrm{W}(9,10,11,12)$} \\
\hline $5 d_{z^{2}}$ & & & 0.12 & \pm 0.05 & 0.17 & 0.07 & 0.08 & 0.06 & 0.08 & -0.12 & -0.11 \\
\hline $5 d_{x k}$ & & & & & & \pm 0.07 & ${ }_{ \pm} 0.13$ & ${ }_{ \pm} 0.19$ & ${ }_{ \pm 0.14}$ & ${ }_{ \pm} 0.24$ & \pm 0.10 \\
\hline $5 \widetilde{d_{x^{2}-y^{2}}}$ & & 0.13 & \pm 0.05 & 0.11 & 0.10 & 0.16 & 0.05 & & \pm 0.20 & 0.05 & -0.14 \\
\hline $5 d_{y z}$ & & & \pm 0.05 & \pm 0.07 & \pm 0.14 & \pm 0.23 & & ${ }_{ \pm} 0.09$ & & & \pm 0.26 \\
\hline $5 d_{x y}$ & & & & \pm 0.12 & & \pm 0.08 & \pm 0.24 & \pm 0.18 & \pm 0.09 & \pm 0.18 & ${ }_{ \pm} 0.08$ \\
\hline $6 s$ & 0.10 & & & & & & & & & & \\
\hline
\end{tabular}


TABLE VI. Totally symmetric molecular orbital for $\mathrm{W}_{9} \mathrm{~N}$ surface molecule where nitrogen is bonded at a $1 \mathrm{CN}$ site.

\begin{tabular}{|c|c|c|c|c|c|c|c|}
\hline $\begin{array}{l}\text { Molecular orbital } \\
\text { Energy }(e \mathrm{~V})\end{array}$ & $\begin{array}{l}\Psi_{6}^{\prime} \\
(-18.33)\end{array}$ & $\begin{array}{l}\Psi_{1}^{\prime} \\
(-12.17)\end{array}$ & $\begin{array}{l}\Psi_{2}^{\prime} \\
(-10.57)\end{array}$ & $\begin{array}{l}\Psi_{3}^{\prime} \\
(-10.38)\end{array}$ & $\begin{array}{l}\Psi_{4}^{\prime} \\
(-9.82)\end{array}$ & $\begin{array}{l}\Psi_{7}^{\prime} \\
(-9.47)\end{array}$ & $\begin{array}{l}\Psi_{5}^{\prime} \\
(-8.43)\end{array}$ \\
\hline \multicolumn{8}{|l|}{$\overline{\mathrm{N}}$} \\
\hline $2 s$ & 0.89 & 0.14 & 0.21 & -0.04 & 0.04 & 0.03 & \\
\hline $\begin{array}{l}2 p_{s} \\
W(1)\end{array}$ & & 0.03 & -0.46 & 0.10 & -0.33 & -0.65 & 0.12 \\
\hline $5 d_{z} 2$ & 0.17 & 0.21 & -0.38 & 0.05 & -0.07 & -0.06 & -0.08 \\
\hline $6 s$ & 0.11 & -0.19 & 0.07 & & & & \\
\hline \multicolumn{8}{|l|}{$\mathrm{W}(2,3,4,5)$} \\
\hline $5 d_{z^{2}}$ & & & 0.21 & 0.16 & 0.07 & -0.25 & 0.08 \\
\hline $5 d_{x}(2,5)$ & & & ${ }_{ \pm 0.12}$ & ${ }_{ \pm} 0.06$ & \pm 0.33 & & \pm 0.05 \\
\hline $5 d_{x^{2}-y^{2}}$ & & & \pm 0.08 & \pm 0.30 & \pm 0.11 & \pm 0.22 & \pm 0.10 \\
\hline $5 d_{y e}(3,4)$ & & & \pm 0.12 & \pm 0.06 & \pm 0.33 & & \pm 0.05 \\
\hline $6 s$ & & -0.18 & -0.08 & & & & \\
\hline \multicolumn{8}{|l|}{$W(6,7,8,9)$} \\
\hline $5 d_{z^{2}}$ & & & -0.10 & -0.19 & & 0.10 & 0.25 \\
\hline $5 d_{x z}$ & & & & +0.11 & & +0.05 & +0.28 \\
\hline $5 d_{y z}$ & & & & \pm 0.11 & & \pm 0.05 & \pm 0.28 \\
\hline $5 d_{x y y}$ & & & \pm 0.10 & & \pm 0.23 & & \pm 0.12 \\
\hline $6 s$ & & -0.12 & & & & & \\
\hline
\end{tabular}

the nitrogen adatom position has a fourfold symmetry (5 $\mathrm{CN}$ or $1 \mathrm{CN}$ sites) rather than a twofold symmetry (2 CN site). ${ }^{10}$ This argument has been explicitly developed in the interpretation of LEED patterns for nitrogen on W(100) by Adams and Germer. ${ }^{9}$ Estrup and Anderson ${ }^{8}$ and Adams and Germer ${ }^{9}$ have chosen the 5 $C N$ site for the nitrogen adatom in the $C(2 \times 2)$ structure, as have Clavenna and Schmidt ${ }^{7}$ for their $\beta_{2}$ nitrogen state on $\mathrm{W}(100)$, which is doubtless equivalent. A second form of high temperature nitrogen, apparently also atomic and very possibly filling in the alternate sites left unfilled in the $C(2 \times 2)$ structure, can be prepared from a low temperature molecularly bound nitro- gen state. ${ }^{7} 11$ As our calculations were performed for an isolated nitrogen adatom, and therefore obviously neglect interaction between adatoms, they are clearly more likely to be pertinent to the $\beta_{2}$ or $C(2 \times 2)$ state than to the $\beta_{1}$ state.

Our calculations indicate that nitrogen prefers the $5 \mathrm{CN}$ site. At this site, the $\sigma$ bond formed by the $2 s$ and $2 p$, atomic orbitals of nitrogen with the atoms of the surface array is not as favorable as at the other sites (Fig. 7); hydrogen also formed a weaker $\sigma$ bond at this site. ${ }^{1}$ But in the case of nitrogen, the $2 p_{z}$ and $2 p_{y}$ atomic orbitals interact more favorably with the tungsten atoms

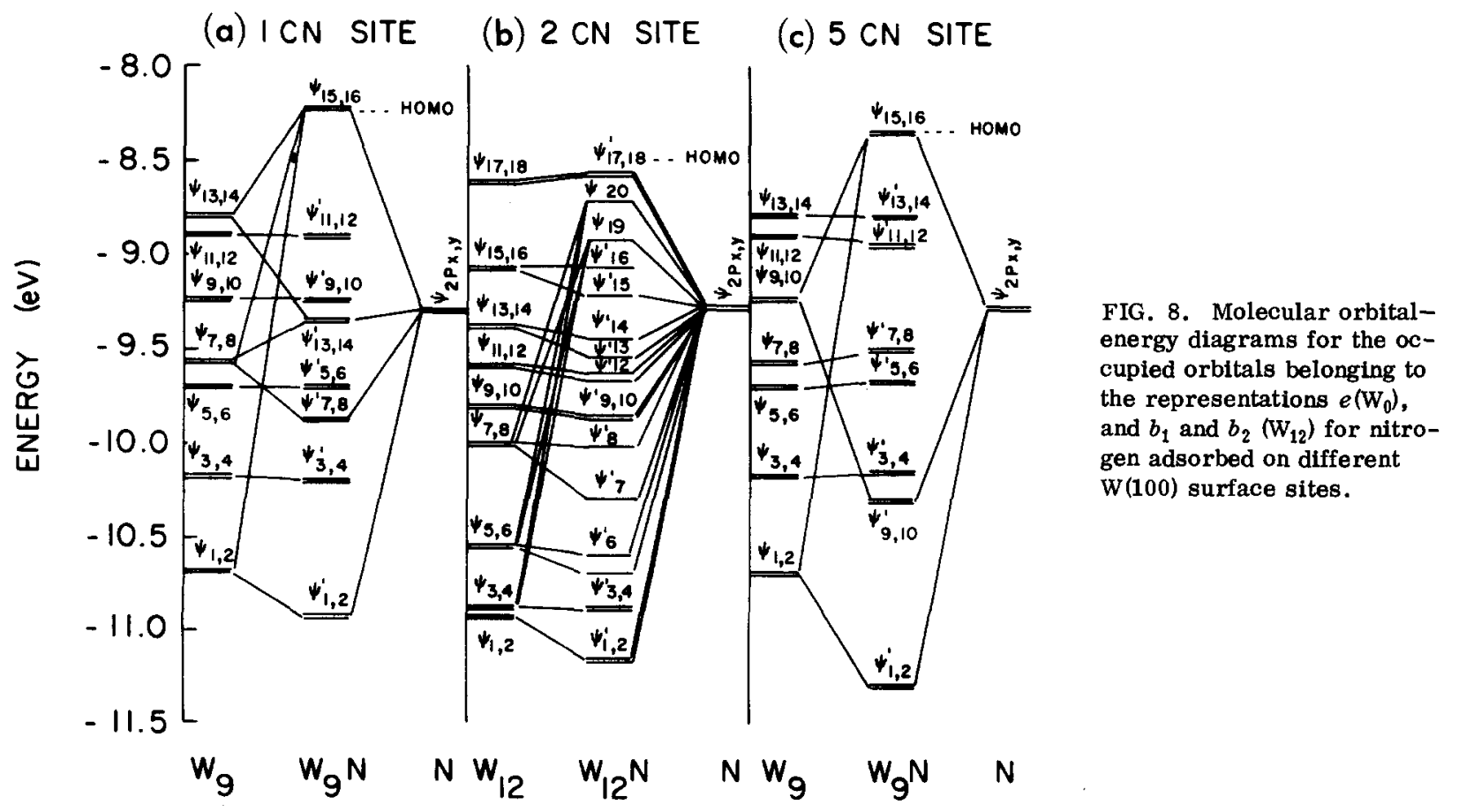


TABLE VII. Totally symmetric molecular orbitals for the $\mathrm{W}_{12} \mathrm{~N}$ surface molecule, where nitrogen is bonded at a $2 \mathrm{CN}$ site.

\begin{tabular}{|c|c|c|c|c|c|c|c|c|c|c|c|c|c|}
\hline $\begin{array}{l}\text { Molecular orbital } \\
\text { Energy (e V) }\end{array}$ & $\Psi_{12}(-18.47)$ & $\begin{array}{l}\boldsymbol{\Psi}_{1}^{\prime} \\
(-12.42)\end{array}$ & $\begin{array}{l}\Psi_{2}^{\prime} \\
\quad(-11 ، 35)\end{array}$ & $\begin{array}{l}\Psi_{3}^{\prime} \\
(-10.98)\end{array}$ & $\begin{array}{l}\Psi_{4}^{\prime} \\
(-10.63)\end{array}$ & $\begin{array}{l}\Psi_{5}^{\prime} \\
(-10.22)\end{array}$ & $\begin{array}{l}\Psi_{6}^{\prime} \\
(-10.03)\end{array}$ & $\begin{array}{l}\Psi_{7}^{\prime} \\
(-9.79)\end{array}$ & $\begin{array}{l}\Psi_{8}^{\prime} \\
(-9.62)\end{array}$ & $\begin{array}{l}\Psi_{y}^{\prime} \\
(-9.33)\end{array}$ & $\begin{array}{l}\Psi_{10}^{\prime} \\
(-8.72)\end{array}$ & $\begin{array}{l}\Psi_{13} \\
(-8.55)\end{array}$ & $\begin{array}{l}\Psi_{11}^{\prime} \\
(-8.26)\end{array}$ \\
\hline \multicolumn{14}{|l|}{$\mathrm{N}$} \\
\hline $2 s$ & -0.86 & -0.17 & 0.05 & -0.05 & 0.10 & -0.16 & 0.05 & 0.03 & 0.08 & & 0.03 & -0.03 & -0.03 \\
\hline $2 p_{z}$ & & 0.02 & 0.18 & -0.26 & 11.26 & -0.47 & 0.05 & 0.34 & 0.16 & -0.32 & -0.01 & 0.28 & -0.17 \\
\hline \multicolumn{14}{|l|}{$W(1,2)$} \\
\hline $5 d_{x^{2}}$ & & & 0.09 & -0.36 & & & -0.07 & 0.07 & -0.11 & 0.07 & & & 0.09 \\
\hline $5 d_{x^{2}-y^{2}}$ & 0.06 & & 0.32 & & -0.06 & -0.14 & & & -0.07 & -0.12 & -0.08 & -0.14 & 0.08 \\
\hline $5 d_{y z}$ & \pm 0.11 & \pm 0.14 & $=0.14$ & \pm 0.08 & $: 0.15$ & \pm 0.17 & $\therefore 0.07$ & $=0.11$ & $\therefore 0.13$ & $=0.09$ & \pm 0.12 & & \pm 0.06 \\
\hline $6 s$ & $\pm 0,09$ & 0.13 & & & & -0.05 & & & & & & & \\
\hline \multicolumn{14}{|l|}{$W(3,4)$} \\
\hline $5 d_{R^{2}}$ & & & & 0.31 & -0.14 & -0.12 & -0.09 & 0.19 & 0.05 & 0.05 & -0.09 & -0.15 & -0.18 \\
\hline $5 \boldsymbol{d}_{x z}$ & & & ${ }_{ \pm} 0.14$ & \pm 0.08 & \pm 0.37 & & & 0.16 & $=0.19$ & $=0.07$ & $=0.20$ & $=0.23$ & $=0.08$ \\
\hline $5 d_{x^{2}-y^{2}}$ & & & -0.31 & -0.12 & -0.10 & -0.14 & 0.11 & -0.06 & & -0.17 & & & 0.16 \\
\hline $6 s^{x^{-2 y-}}$ & & 0.18 & & & & -0.0 .5 & & & & & & & \\
\hline \multicolumn{14}{|l|}{$W(5,6,7,8)$} \\
\hline $5 d_{z^{2}}$ & & & & 0.09 & & -0.17 & 0.1 .1 & -0.10 & & & -0.12 & & 0.14 \\
\hline $5 d_{x z}$ & & & & \pm 0.05 & & $\pm 0: 16$ & \pm 0.17 & 0.10 & & $=0.19$ & $=0,05$ & .0 .29 & $=0.13$ \\
\hline $5 d x^{2}-y^{2}$ & & & -0.12 & & -0.09 & & -0.20 & 0.08 & -0.08 & $-0,22$ & & & -0.17 \\
\hline $5 d_{y z-y}$ & & & $=0.05$ & $\pm 0.0 \overline{5}$ & & & & $0.1 \overline{0}$ & 0.08 & $=0.20$ & \pm 0.25 & & $=0.10$ \\
\hline $5 d_{\mathrm{wz}}$ & & & & & \pm 0.11 & & \pm 0.08 & $=0.07$ & $=0.30$ & +0.11 & 0.14 & $=0.06$ & $=0.05$ \\
\hline $6 s$ & & 0.12 & & & & 0.05 & & & & & -0.05 & & \\
\hline \multicolumn{14}{|l|}{$W(9,10,11,12)$} \\
\hline $5 d_{\mathbf{z}^{2}}$ & & & & -0.10 & & 0.16 & & & 0.08 & -0.09 & -0.10 & -0.16 & \\
\hline $5 d_{x z}$ & & & -0.06 & & & & \pm 0.06 & $=0.17$ & $=0.17$ & & 0.21 & $=0.14$ & $=0.1 \overline{5}$ \\
\hline $5 d_{x^{2}-y^{2}}$ & & & 0.11 & 0.69 & & 0.06 & 0.13 & 0.07 & & -0.11 & 0.10 & -0.22 & -0.08 \\
\hline $5 d_{y z}$ & & & & & & & \pm 0.26 & -0.08 & $=0.17$ & & & & $=0.31$ \\
\hline$-j d_{x y}$ & & & & & & \pm 0.117 & & $\therefore 0.22$ & & .0 .08 & $=0.20$ & ${ }_{ \pm} 0.07$ & -0.12 \\
\hline $6 s y$ & & 0.10 & & & & & & & & & & & \\
\hline
\end{tabular}


TABLE VIII. Totally symmetric molecular orbitals for the $\mathrm{W}_{9} \mathrm{~N}$ surface molecule where nitrogen is bonded at a $5 \mathrm{CN}$ site.

\begin{tabular}{|c|c|c|c|c|c|c|c|}
\hline $\begin{array}{l}\text { Molecular orbital } \\
\text { Energy (eV) }\end{array}$ & $\begin{array}{l}\Psi_{6} \\
(-18.71)\end{array}$ & $\begin{array}{l}\Psi_{1}^{\prime} \\
(-11.97)\end{array}$ & $\begin{array}{l}\Psi_{2}^{\prime} \\
(-10.70)\end{array}$ & $\begin{array}{l}\Psi_{3}^{\prime} \\
(-10,36)\end{array}$ & $\begin{array}{l}\Psi_{4}^{\prime} \\
(-9.39)\end{array}$ & $\begin{array}{l}\Psi_{5}^{\prime} \\
(-8.61)\end{array}$ & $\begin{array}{l}\Psi_{7}^{\prime} \\
(-7.49)\end{array}$ \\
\hline \multicolumn{8}{|l|}{$\mathrm{N}$} \\
\hline $2 s$ & -0.82 & 0.11 & 0.10 & & 0.17 & -0.09 & 0.06 \\
\hline $\begin{array}{l}2 p_{z} \\
W(1)\end{array}$ & & -0.11 & 0.56 & -0.25 & 0.43 & 0.02 & -0.21 \\
\hline $5 d_{z^{2}}$ & -0.12 & 0.28 & -0.23 & -0.09 & -0.31 & & 0.35 \\
\hline $6 s$ & -0.06 & -0.21 & & & & & 0.08 \\
\hline \multicolumn{8}{|l|}{$\mathrm{W}(2,3,4,5)$} \\
\hline $5 d_{x^{2}}$ & & & 0.15 & 0.17 & 0.13 & -0.07 & 0.22 \\
\hline $5 d_{x z}(2,5)$ & & & \pm 0.21 & \pm 0.18 & \pm 0.16 & & \pm 0.21 \\
\hline $5 d x^{2}-y^{2}$ & \pm 0.06 & & \pm 0.08 & \pm 0.24 & & & \pm 0.17 \\
\hline $5 d_{y z}(3,4)$ & & & \pm 0.21 & \pm 0.18 & \pm 0.16 & & \pm 0.21 \\
\hline $6 s$ & -0.05 & -0.14 & & & & & \\
\hline \multicolumn{8}{|l|}{$\mathrm{W}(6,7,8,9)$} \\
\hline $5 d_{z^{2}}$ & & & -0.11 & -0.19 & 0.08 & 0.24 & \\
\hline $5 d_{x z}$ & & & \pm 0.05 & \pm 0.07 & \pm 0.06 & \pm 0.30 & \\
\hline $5 d_{y z}$ & & & \pm 0.05 & \pm 0.07 & \pm 0.06 & \pm 0.30 & \\
\hline $5 d_{x y}$ & & & \pm 0.06 & \pm 0.10 & \pm 0.29 & & \pm 0.35 \\
\hline $6 s$ & & -0.13 & & & -0.09 & & \\
\hline
\end{tabular}

surrounding the $5 \mathrm{CN}$ site (Fig. 8) than at the other sites, and this factor dominates the selection of the 5 $\mathrm{CN}$ site for chemisorption. As Table XIII shows, these atomic orbitals are able to form the lowest energy molecular orbitals of $e$ symmetry at the $5 \mathrm{CN}$ site because of their overlap with the appropriate $d$ orbitals of the four surrounding tungsten atoms.

Further comments are suggested by the data presented in Table III. The bond energy of $3.25 \mathrm{eV}$ calculated for the $5 \mathrm{CN}$ site is about half the correct value
(6. $6 \mathrm{eV}$ ); the EHMO is characteristically unreliable for absolute (less so for relative) energies. ${ }^{2}$ The $2.05 \AA$ distance between the nitrogen atom and the tungsten atom underlying it corresponds to a Pauling ${ }^{5}$ bond order of 0.85 ; the distance between the nitrogen atom and each of the other four tungsten atoms to which it is coordinated is $2.28 \AA$ with an indicated bond order 0.4 , so that in all the calculation suggests a bond order of 2.45 between the nitrogen atom and the tungsten surface. Table III also shows the total bond overlap population, as defined by Mulliken, ${ }^{12}$ for all bonds between nitrogen and tungsten

TABLE IX. Molecular orbitals which belong to the $e$ representation in the $C_{4 v}$ symmetry group for the $\mathrm{W}_{9}$ surface arrays representing the $1 \mathrm{CN}$ and $5 \mathrm{CN}$ sites.

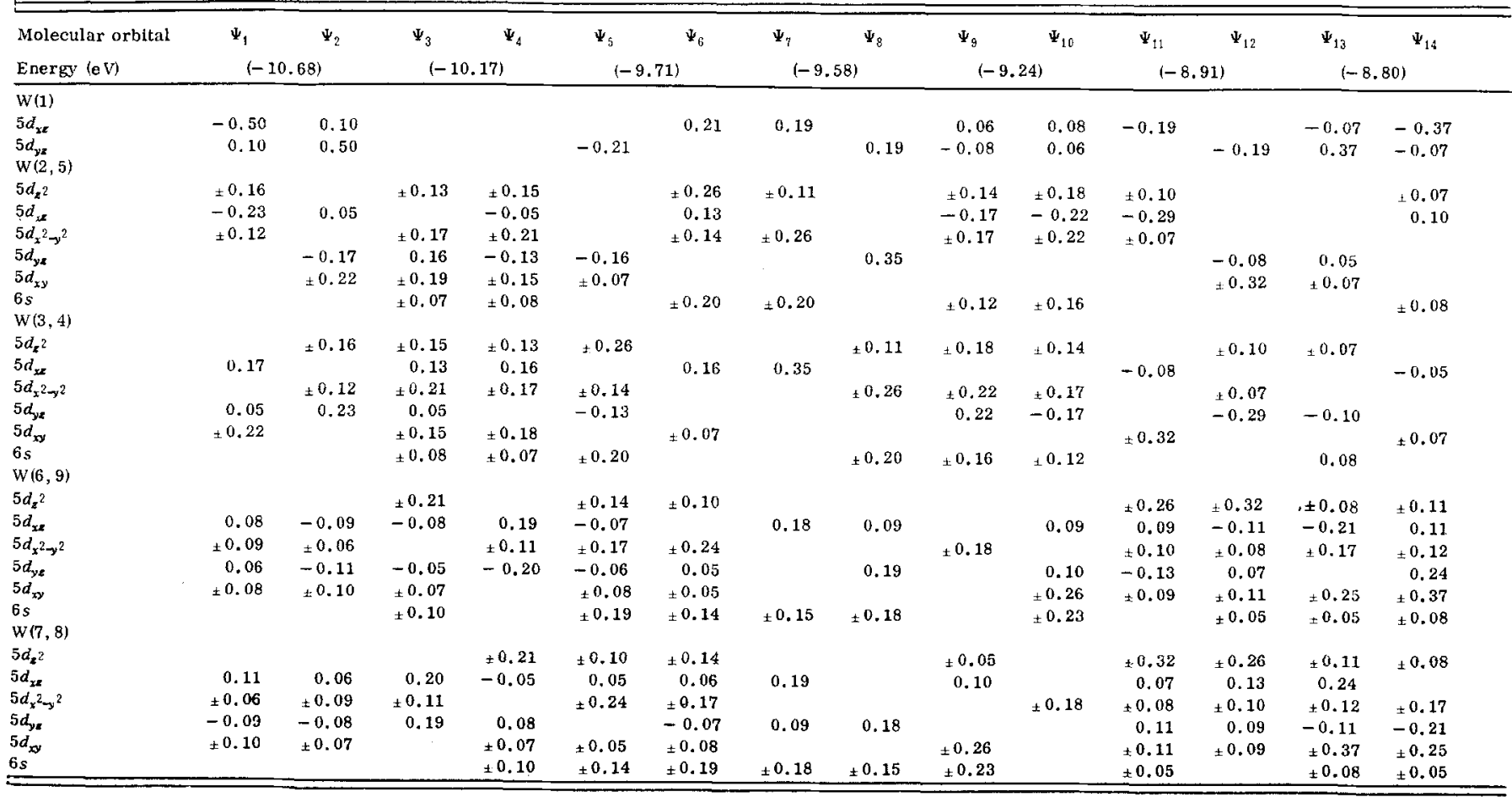


TABLE X. Molecular orbitals which belong to the $b_{1}$ and $b_{2}$ representations in the $C_{2 v}$ symmetry group for the $\mathrm{W}_{12}$ surface array representing the $2 \mathrm{CN}$ site.

\begin{tabular}{|c|c|c|c|c|c|c|c|c|c|}
\hline $\begin{array}{l}\text { Molecular orbital } \\
\text { Energy (eV) }\end{array}$ & $\begin{array}{l}\Psi_{1} \\
(-10.92) \\
\end{array}$ & $\begin{array}{l}\boldsymbol{\Psi}_{2} \\
(-10.92) \\
\end{array}$ & $\begin{array}{l}\boldsymbol{\Psi}_{3} \\
(-10.88)\end{array}$ & $\begin{array}{l}\Psi_{1} \\
(-10.88)\end{array}$ & $\begin{array}{l}\Psi \\
(-10.56)\end{array}$ & $\begin{array}{l}\Psi_{i i} \\
(-10.56)\end{array}$ & $\begin{array}{l}\Psi \\
(-10.01)\end{array}$ & $\begin{array}{l}\Psi_{B} \\
(-10,01)\end{array}$ & $\begin{array}{l}\Psi_{9} \\
(-0.80)\end{array}$ \\
\hline \multicolumn{10}{|l|}{$w(1,2)$} \\
\hline $5 d_{z^{2}}$ & +0.18 & +0.15 & & & .0 .111 & & & & \pm 0.18 \\
\hline $5 d_{x z}$ & -0.17 & 0.21 & -0.06 & & & -0.68 & -0.10 & & 0.17 \\
\hline $5 d_{x^{2}-y^{2}}$ & ${ }_{ \pm .0 .17}$ & 0.14 & $=0.05$ & +0.09 & \pm 0.11 & & & 0.05 & \\
\hline $5 d_{y x}$ & & & & & -11.23 & -0.05 & 0.08 & -0.23 & \\
\hline $5 d_{x y}$ & . 0.12 & 0.14 & $=0.14$ & \pm 0.08 & \pm 0.08 & \pm 0.37 & & & \\
\hline $6 s$ & & & & $=0.18$ & +0.08 & & & & \\
\hline$W(3,4)$ & & & & & & & & & \\
\hline $5 d_{\mathbf{z}^{2}}$ & \pm 0.15 & ${ }_{+} 0.18$ & & & & +0.10 & & & ${ }_{1} 0.19$ \\
\hline $5 d_{x z}$ & & & & & 0.05 & -0.23 & 0.23 & 0.08 & \\
\hline $5 d_{x^{2}-y^{2}}$ & $\div 0.14$ & -0.17 & \pm 0.09 & \pm 0.05 & & t. 0.11 & \pm 0.05 & & \\
\hline $5 d_{y z}$ & -0.21 & -0.17 & & -0.06 & \pm 0.08 & & & 0.10 & 0.15 \\
\hline $5 d_{x y}$ & \pm 0.14 & .0 .12 & ${ }_{ \pm} 0.08$ & 0.14 & $=0.37$ & $=0.08$ & & & \\
\hline $6 s$ & & & \pm 0.18 & \pm 0.09 & & -0.08 & & & \\
\hline$w(5,8)$ & & & & & & & & & \\
\hline $5 d_{z_{2}}$ & \pm 0.09 & & & & & $=0.12$ & \pm 0.07 & & \pm 0.27 \\
\hline $5 d_{x z}$ & & & & & -0.11 & & & +0.23 & \\
\hline $5 d x^{2}-y^{2}$ & & ${ }_{ \pm} 0.17$ & & & -0.11 & & \pm 0.08 & & \\
\hline $5 d_{y z}$ & & 0.15 & & & & & 0.09 & & 0.08 \\
\hline $5 d_{x y}$ & ${ }_{ \pm} 0.08$ & & & & .0 .07 & & & \pm 0.30 & \pm 0.07 \\
\hline $6 s$ & & & \pm 0.23 & 0.16 & 0.12 & & & & \\
\hline$W(6,7)$ & & & & & & & & & \\
\hline $5 d_{k^{2}}$ & & \pm 0.09 & & & \pm 0.07 & \pm 0.10 & 0.09 & & \pm 0.14 \\
\hline $5 d_{x z}$ & & & & & 0.10 & 0.06 & -0.17 & 0.16 & \pm 0.08 \\
\hline $5 d^{2} x^{2}+y^{2}$ & \pm 0.17 & & & & +0.10 & & \pm 0.08 & & \\
\hline $5 d_{y z}$ & 0.15 & & & & & & -0.06 & -0.07 & -0.17 \\
\hline $5 d_{x y}$ & & 0.08 & & & 10.09 & & \pm 0.19 & 0.23 & \pm 0.09 \\
\hline $6 s$ & & & & +0.28 & +0.10 & & & & \\
\hline$W(9,12)$ & & & & & & & & & \\
\hline $5 d_{z^{2}}$ & ${ }_{ \pm} 0.09$ & & & & $=0.10$ & ${ }_{\perp} 0.07$ & & 0.09 & : 0.28 \\
\hline $5 d_{x z}$ & & -0.15 & & & & & 0.07 & -0.07 & 0.06 \\
\hline $5 d_{x^{2}}^{2} y^{2}$ & & \pm 0.17 & & & & \pm 0.10 & & \pm 0.08 & \\
\hline $5 d_{y x}$ & & & & & & -0.10 & 0.16 & 0.17 & \\
\hline $5 d_{x y}$ & \pm 0.08 & & & & & \pm 0.09 & 0.23 & +0.19 & ×0.06 \\
\hline $6 s$ & & & \pm 0.28 & & \pm 0.08 & \pm 0.10 & & & \\
\hline$W(10,11)$ & & & & & & & & & \\
\hline $5 d_{z^{2}}$ & & \pm 0.09 & & & ${ }_{ \pm} 0.12$ & & & .0 .07 & \pm 0.16 \\
\hline $5 d_{x z}$ & 0.15 & & & & & & & 0.09 & -0.16 \\
\hline $5 d_{x^{2}-y^{2}}^{2}$ & \pm 0.17 & & & & & \pm 0.11 & & ${ }_{ \pm} 0.08$ & \\
\hline $5 d_{y \varepsilon}$ & & & & & & 0.11 & -0.23 & & -0.09 \\
\hline $5 d_{x y}$ & & \pm 0.08 & & & & +0.07 & ${ }_{ \pm} 0.30$ & & \pm 0.09 \\
\hline $6 s$ & & & \pm 0.16 & \pm 0.23 & & \pm 0.12 & & & \\
\hline $\begin{array}{l}\text { Molecular orbital } \\
\text { Energy (e V) }\end{array}$ & $\begin{array}{l}\Psi_{10} \\
(-9.80) \\
\end{array}$ & $\begin{array}{l}\Psi_{11} \\
(-9.59) \\
\end{array}$ & $\begin{array}{l}\Psi_{12} \\
(-9.59) \\
\end{array}$ & $\begin{array}{l}\boldsymbol{\Psi}_{13} \\
(-9.38) \\
\end{array}$ & $\begin{array}{l}\Psi_{14} \\
(-9.38) \\
\end{array}$ & $\begin{array}{l}\Psi_{15} \\
(-9.07)\end{array}$ & $\begin{array}{l}\Psi_{16} \\
(-9.07)\end{array}$ & $\begin{array}{l}\Psi_{18} \\
(-8.62)\end{array}$ & $\begin{array}{l}\Psi_{18} \\
(-8.62)\end{array}$ \\
\hline$W(1,2)$ & & & & & & & & & \\
\hline $5 d_{z^{2}}$ & \pm 0.19 & \pm 0.09 & & & & & & & \\
\hline $5 d_{x x}$ & -0.15 & & \pm 0.07 & ${ }_{ \pm 0} 0.10$ & ${ }_{ \pm 0.08}$ & & & \pm 0.14 & \pm 0.05 \\
\hline $5 d d_{x^{2}-y^{2}}^{2}$ & & & & 0.20 & -0.24 & -0.13 & & & -0.08 \\
\hline $5 d_{y z}$ & & & & ${ }_{ \pm 0.12}$ & \pm 0.10 & & & \pm 0.27 & \pm 0.10 \\
\hline $5 d^{9 / 2}$ & & 0.14 & 0.11 & -0.19 & -0.16 & & & 0.05 & \\
\hline $6 s$ & & & & ${ }_{ \pm} 0.09$ & \pm 0.11 & \pm 0.20 & & & \pm 0.11 \\
\hline$W(3,4)$ & & & & & & & & & \\
\hline $5 d_{z^{2}}$ & & & & & & & & & \\
\hline $5 d_{x x}$ & \pm 0.18 & ${ }_{ \pm} 0.07$ & \pm 0.09 & ${ }_{ \pm 0.08}$ & \pm 0.10 & & & \pm 0.05 & \pm 0.14 \\
\hline $5 d_{x^{2}}^{2} y^{2}$ & & 0.11 & -0.14 & 0.16 & -0.19 & & & & -0.05 \\
\hline $5 d_{y z}$ & & & & ${ }_{ \pm 0.10}$ & \pm 0.12 & & & \pm 0.10 & \pm 0.27 \\
\hline $5 d_{x y}$ & 0.17 & & & -0.24 & -0.20 & & -0.13 & 0.08 & \\
\hline $6 s$ & & & & \pm 0.11 & ${ }_{1} 0.09$ & & +0.20 & \pm 0.11 & \\
\hline$W(5,8)$ & & & & & & & & & \\
\hline $5 d_{z^{2}}$ & & & & & & & & & \\
\hline $5 d_{x z}$ & \pm 0.16 & ${ }_{ \pm} 0.09$ & 0.08 & & & \pm 0.09 & \pm 0.14 & \pm 0.14 & \\
\hline $5 d_{x^{2}}^{2} \gamma^{2}$ & 0.09 & & -0.18 & 0.11 & 0.09 & -0.14 & -0.27 & -0.18 & -0.08 \\
\hline $5 d_{y z}$ & & \pm 0.21 & \pm 0.18 & ${ }_{ \pm 0} 0.18$ & \pm 0.12 & \pm 0.14 & ${ }_{ \pm} 0.09$ & & \pm 0.13 \\
\hline $5 d_{x y}^{\prime 2}$ & \pm 0.16 & 0.18 & 0.15 & -0.10 & & & -0.13 & -0.24 & -0.18 \\
\hline $6 s$ & \pm 0.09 & \pm 0.08 & \pm 0.08 & & \pm 0.20 & $\$ 0.07$ & +0.18 & & \pm 0.15 \\
\hline$w(6,7)$ & & & & & & & & & \\
\hline $5 d_{x^{2}}$ & & & & & & & & & \\
\hline $5 d_{x z}$ & \pm 0.28 & ${ }_{ \pm} 0.06$ & \pm 0.11 & & & \pm 0.10 & \pm 0.13 & \pm 0.12 & \pm 0.07 \\
\hline $5 d_{x^{2}-y^{2}}^{2}$ & & 0.17 & -0.07 & -0.11 & -0.09 & -0.17 & 0.26 & 0.09 & -0.18 \\
\hline $5 d_{y z}$ & & \pm 0.23 & ${ }_{ \pm} 0.15$ & $\frac{1}{ \pm} 0.15$ & \pm 0.16 & +0.13 & ${ }_{ \pm} 0.11$ & ${ }_{ \pm} 0.09$ & ${ }_{ \pm} 0.10$ \\
\hline $5 d_{x y}$ & 0.06 & 0.19 & 0.13 & & +0.10 & 0.06 & -0.12 & -0.07 & 0.29 \\
\hline $6 s^{3}$ & \pm 0.06 & ${ }_{ \pm} 0.05$ & \pm 0.09 & ${ }_{1} 0.19$ & & & \pm 0.19 & \pm 0.08 & \pm 0.13 \\
\hline $\mathrm{W}(9,12)$ & & & & & & & & & \\
\hline $5 d_{x^{2}}$ & & & & & & & & & \\
\hline $5 d_{x z}$ & \pm 0.14 & \pm 0.11 & \pm 0.06 & & & \pm 0.13 & \pm 0.10 & ${ }_{ \pm} 0.07$ & \pm 0.12 \\
\hline $5 d_{x^{2}-y^{2}}$ & 0.17 & 0.13 & -0.19 & 0.10 & -0.15 & -0.12 & -0.06 & 0.29 & 0.07 \\
\hline $5 d_{y, z}$ & & ${ }_{ \pm} 0.15$ & \pm 0.23 & \pm 0.16 & \pm 0.11 & \pm 0.11 & \pm 0.13 & ${ }_{ \pm 0} 0.10$ & \pm 0.09 \\
\hline $5 d_{x y}$ & -0.08 & 0.07 & 0.17 & -0.09 & -0.19 & -0.26 & -0.17 & 0.18 & 0.09 \\
\hline $6 s$ & \pm 0.09 & \pm 0.09 & & & & \pm 0.19 & & ${ }_{ \pm 0} 0.13$ & \pm 0.08 \\
\hline $\mathrm{W}(10,11)$ & & & & & & & & & \\
\hline $5 d_{x^{2}}$ & & & & & & & & & \\
\hline $5 d_{x z}$ & \pm 0.27 & \pm 0.08 & \pm 0.09 & & & \pm 0.14 & \pm 0.09 & & \pm 0.14 \\
\hline $5 d_{x^{2}-y^{2}}$ & -0.08 & 0.15 & -0.18 & & -0.10 & -0.13 & & -0.18 & 0.24 \\
\hline $5 d_{y z}$ & & \pm 0.18 & \pm 0.21 & \pm 0.12 & \pm 0.18 & \pm 0.09 & \pm 0.14 & \pm 0.13 & \\
\hline $5 d_{x y}$ & & 0.18 & & 0.09 & -0.11 & 0.27 & -0.14 & 0.08 & -0.18 \\
\hline $6 s^{2}$ & \pm 0.07 & \pm 0.08 & \pm 0.08 & \pm 0.20 & & \pm 0.18 & -0.07 & \pm 0.15 & \\
\hline
\end{tabular}


TABLE XI. Molecular orbitals which belong to the $e$ representation for the $\mathrm{W}_{9} \mathrm{~N}$ surface molecule where nitrogen is bonded at a $1 \mathrm{CN}$ site.

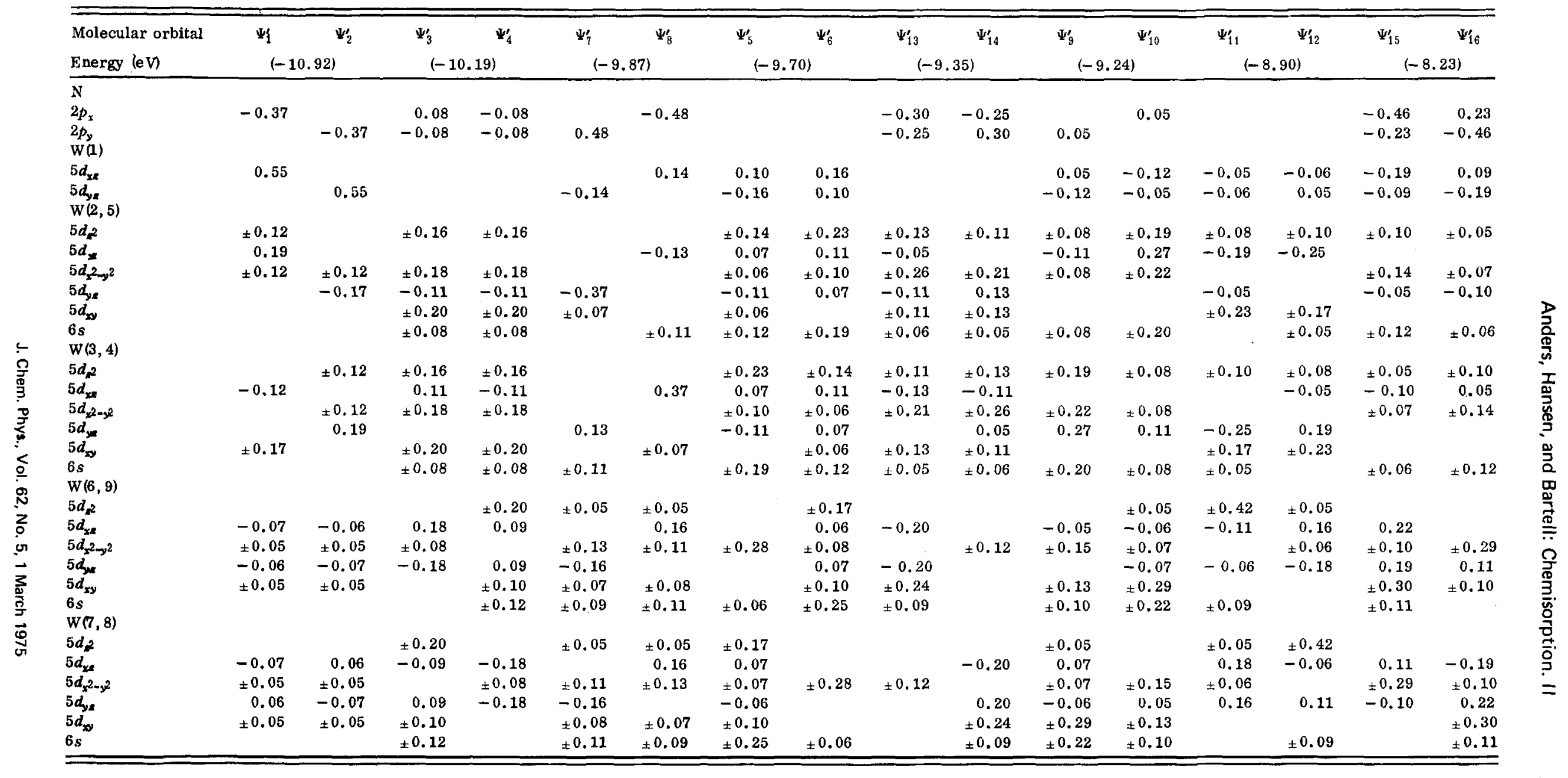


TABLE XII. Molecular orbitals which belong to the $b_{1}$ and $b_{2}$ representations for the $W_{12} \mathrm{~N}$ surface molecule where nitrogen is bonded at a $2 \mathrm{CN}$ site.

\begin{tabular}{|c|c|c|c|c|c|c|c|c|c|c|}
\hline $\begin{array}{l}\text { Molecular orbital } \\
\text { Energy (eV) }\end{array}$ & $\begin{array}{l}\Psi_{1}^{\prime} \\
\quad(-11.18) \\
\end{array}$ & $\begin{array}{l}\Psi_{2}^{\prime} \\
(-11.14)\end{array}$ & $\begin{array}{l}\Psi_{3}^{\prime} \\
(-10.90)\end{array}$ & $\begin{array}{l}\Psi_{1}^{\prime} \\
(-10.89)\end{array}$ & $\begin{array}{l}\Psi^{\prime} \\
(-10.70)\end{array}$ & $\begin{array}{l}\Psi_{6}^{\prime} \\
(-10.63)\end{array}$ & $\begin{array}{l}\Psi_{?}^{\prime} \\
(-10.28)\end{array}$ & $\begin{array}{l}\Psi_{8}^{\prime} \\
(-10.02)\end{array}$ & $\begin{array}{l}\Psi_{9}^{\prime} \\
(-9.86)\end{array}$ & $\begin{array}{l}\boldsymbol{\Psi}_{10}^{\prime} \\
(-9.84)\end{array}$ \\
\hline \multicolumn{11}{|l|}{$\mathrm{N}$} \\
\hline $2 p_{x}$ & 0.35 & & -0.03 & & & 0.10 & & -0.08 & & -0.22 \\
\hline \multicolumn{11}{|l|}{$w(1,2)$} \\
\hline $5 d_{s^{i}}$ & & \pm 0.23 & & & 0.14 & & 0.13 & & +0.22 & \\
\hline $5 d_{x z}$ & 0.19 & & 0.21 & & & -0.09 & & -0.13 & & -0.24 \\
\hline $5 d x^{2}-y^{2}$ & & \pm 0.17 & & 10.16 & & & \pm 0.16 & & \pm 0.05 & \\
\hline $5 d_{y z}$ & & 0.21 & & -0.05 & 0.27 & & -0.14 & & 0.06 & \\
\hline $5 d_{x y}$ & \pm 0.28 & & & & & +0.31 & & \pm 0.07 & & 0.05 \\
\hline $6 s$ & & $=0.06$ & & \pm 0.20 & & & \pm 0.07 & & & \\
\hline \multicolumn{11}{|l|}{$W(3,4)$} \\
\hline $5 d_{z^{2}}$ & \pm 0.18 & & \pm 0.11 & & & & & \pm 0.07 & & \pm 0.27 \\
\hline $5 d_{x z}$ & & & 0.07 & & & -0.22 & & 0.23 & & -0.06 \\
\hline $5 d_{x^{2}-y^{2}}$ & \pm 0.08 & & \pm 0.22 & & & .0 .13 & & \pm 0.05 & & \\
\hline $5 d_{y z}$ & & 0.19 & & -0.12 & -0.09 & & 0.19 & & 0.11 & \\
\hline $5 d_{n y}$ & & \pm 0.07 & & $\pm 0,11$ & \pm 0.40 & & 10.16 & & & \\
\hline $6 s$ & \pm 0.10 & & \pm 0.16 & & & 0.11 & & & & \\
\hline \multicolumn{11}{|l|}{$W(5,6,7,8)$} \\
\hline $5 d_{z^{2}}$ & & \pm 0.07 & +0.07 & & & 0.11 & & +0.08 & 1.0 .20 & \pm 0.07 \\
\hline $5 d_{x z}$ & 0.05 & & & & \pm 0.10 & & & -0.10 & 10.23 & 0.07 \\
\hline $5 d_{x^{2}-y^{2}}$ & \pm 0.05 & $\$ 0.10$ & \pm 0.12 & & & & 0.11 & \pm 0.07 & & \\
\hline $5 d_{y \varepsilon}$ & \pm 0.08 & \pm 0.06 & & 0.06 & 0.07 & & -0.10 & \pm 0.08 & -0.08 & \pm 0.13 \\
\hline $5 d_{x y}$ & & \pm 0.08 & & & \pm 0.07 & & $: 0.19$ & \pm 0.11 & \pm 0.13 & \pm 0.08 \\
\hline $6 s$ & \pm 0.05 & \pm 0.08 & +0.10 & \pm 0.25 & & 0.07 & 0.10 & & & \\
\hline \multicolumn{11}{|l|}{$W(9,10,11,12)$} \\
\hline $5 d_{z^{2}}$ & & \pm 0.05 & \pm 0.05 & \pm 0.05 & +0.05 & .0 .06 & 0.12 & \pm 0.05 & \pm 0.08 & +0.27 \\
\hline $5 d_{x z}$ & -0.06 & \pm 0.06 & 0.09 & & +0.10 & & & & ¿ 0.19 & \\
\hline $5 d_{x^{2}-y^{2}}$ & \pm 0.05 & \pm 0.07 & \pm 0.08 & \pm 0.09 & +0.05 & 10.13 & $: 0.10$ & & & \pm 0.08 \\
\hline $5 d_{y z}$ & & & & & 0.06 & $=0.10$ & & \pm 0.19 & -0.10 & +0.14 \\
\hline $5 d_{x y}$ & \pm 0.07 & & & & \pm 0.05 & & & \pm 0.29 & & \\
\hline $6 s$ & \pm 0.13 & & \pm 0.19 & \pm 0.12 & & 0.15 & & & & \\
\hline $\begin{array}{l}\text { Molecular orbital } \\
\text { Energy (ev) }\end{array}$ & $\begin{array}{l}\Psi_{11} \\
(-9.67)\end{array}$ & $\begin{array}{l}\Psi_{12}^{\prime} \\
(-9,61)\end{array}$ & $\left.\Psi_{13}^{\prime}-9.55\right)$ & $\Psi_{14}^{\prime}(-9.46)$ & $\begin{array}{l}\boldsymbol{w}_{15} \\
(-9,23)\end{array}$ & $\begin{array}{l}\Psi_{16}^{\prime} \\
(-9.07)\end{array}$ & $\begin{array}{l}\Psi_{19} \\
(-8.93)\end{array}$ & $\Psi_{210}(-8.72)$ & $\begin{array}{l}\Psi_{17}^{\prime} \\
(-8.58)\end{array}$ & $\begin{array}{l}\Psi_{18}^{\prime} \\
(-8.56)\end{array}$ \\
\hline \multicolumn{11}{|l|}{$\bar{N}$} \\
\hline $2 p_{x}$ & & -0.16 & & -0.31 & 0.34 & & & -0.53 & 0.34 & \\
\hline $\begin{array}{l}2 p_{y} \\
W(1,2)\end{array}$ & -0.21 & & -0.15 & & & 0.02 & 0.44 & & & -0.20 \\
\hline $5 d_{z^{2}}$ & \pm 0.12 & & \pm 0.13 & & & & & & & \pm 0.11 \\
\hline $5 d_{x z}$ & & & & -0.23 & -0.10 & & & 0.14 & & \\
\hline $5 \sqrt[d_{x^{2}}-y^{2}]{2}$ & & & \pm 0.05 & & & & & & & \pm 0.23 \\
\hline $5 d_{y z}$ & 0.14 & & -0.05 & & & & 0.22 & & & 0.06 \\
\hline $5 d_{x y}$ & & \pm 0.07 & & \pm 0.10 & 10.19 & & & \pm 0.06 & \pm 0.15 & \\
\hline \multirow{2}{*}{\multicolumn{11}{|c|}{$\begin{array}{l}6 s \\
W(3,4)\end{array}$}} \\
\hline & & & & & & & & & & \\
\hline $5 d_{x^{2}}$ & & \pm 0.14 & & \pm 0.06 & \pm 0.12 & & & & ${ }_{ \pm} 0.20$ & \\
\hline $5 d_{x x}$ & & 0.11 & & -0.30 & -0.05 & & & & 0.06 & \\
\hline $5 d_{x^{2}-y^{2}}$ & & & & \pm 0.16 & ${ }_{ \pm} 0.05$ & & & ${ }_{ \pm} 0.16$ & \pm 0.23 & \\
\hline $5 d_{y z}$ & 0.22 & & 0.21 & & & -0.11 & 0.27 & & & \\
\hline $5 d_{x y}$ & & & \pm 0.10 & & & \pm 0.21 & \pm 0.15 & & & \pm 0.07 \\
\hline \multicolumn{11}{|l|}{$6 s$} \\
\hline \multicolumn{11}{|l|}{$W(5,6,7,8)$} \\
\hline $5 d_{s^{2}}$ & \pm 0.20 & \pm 0.14 & \pm 0.06 & & \pm 0.05 & \pm 0.12 & \pm 0.21 & \pm 0.09 & \pm 0.09 & \pm 0.05 \\
\hline $5 d_{x \varepsilon}$ & \pm 0.15 & 0.14 & & -0.06 & 0.10 & \pm 0.27 & \pm 0.08 & 0.16 & 0.08 & \pm 0.13 \\
\hline $5 d_{x^{2}-y^{2}}$ & & & \pm 0.36 & \pm 0.06 & ${ }_{ \pm} 0.09$ & ${ }_{ \pm} 0.10$ & & \pm 0.13 & \pm 0.08 & \\
\hline $5 d_{y z}$ & 0.13 & \pm 0.05 & -0.15 & \pm 0.11 & & -0.12 & 0.10 & \pm 0.28 & \pm 0.12 & -0.22 \\
\hline $5 d_{x y}$ & \pm 0.07 & \pm 0.11 & & \pm 0.12 & ${ }_{ \pm} 0.08$ & ${ }_{ \pm} 0.20$ & \pm 0.20 & \pm 0.08 & \pm 0.23 & \pm 0.12 \\
\hline \multirow{2}{*}{\multicolumn{11}{|c|}{$\mathrm{W}(9,10,11,12)$}} \\
\hline & & & & & & & & & & \\
\hline $5 d_{k^{2}}$ & \pm 0.10 & \pm 0.09 & \pm 0.08 & \pm 0.11 & ${ }_{ \pm} 0.12$ & ${ }_{ \pm} 0.09$ & \pm 0.06 & \pm 0.11 & ${ }_{ \pm} 0.10$ & \pm 0.08 \\
\hline $5 d_{2 s}$ & & 0.17 & & -0.21 & 0.10 & \pm 0.06 & \pm 0.07 & & -0.18 & \pm 0.22 \\
\hline $5 d_{x^{2}-y^{2}}$ & & $\pm 0,30$ & & \pm 0.12 & ${ }_{ \pm} 0.07$ & ${ }_{ \pm 0} 0.14$ & \pm 0.05 & ${ }_{ \pm} 0.08$ & \pm 0.10 & ${ }_{ \pm} 0.12$ \\
\hline $5 d_{y z}$ & 0.11 & \pm 0.09 & -0.11 & \pm 0.06 & \pm 0.27 & -0.15 & & & \pm 0.17 & 0.16 \\
\hline $5 d_{x y}$ & \pm 0.23 & & & & ${ }_{ \pm} 0.20$ & \pm 0.05 & \pm 0.06 & \pm 0.08 & & \pm 0.19 \\
\hline $6 s$ & & & & +0.05 & & & & & & \\
\hline
\end{tabular}

atoms; the number is greater for the $5 \mathrm{CN}$ than for the $2 \mathrm{CN}$ and $1 \mathrm{CN}$ positions, and by this criterion the total bond order is even greater than for nitrogen in the WN diatomic molecule (calculated bond overlap population 0.852 ).

Table III shows a negative charge on the nitrogen in the $5 \mathrm{CN}$ position (less negative than for either of the other two sites considered). We believe that any reasonable parametrization of the calculation will lead to a negative charge on the nitrogen in any configuration on any crystal face. This suggests that nitrogen adsorption should lead to formation of a double layer negative out, and hence to an increase in work function. Delchar and Ehrlich $^{b}$ have obtained careful contact potential data on this subject for (100), (111), and (110) faces of tungsten, and have reviewed field emission work function data of others for these and a number of other crystal faces. On most crystal faces, nitrogen adsorption leads to an increase in work function as the negative charge on nitrogen suggests, but on the (100) face nitrogen chemisorption results in a lowered work function. Surely the 
TABLE XIII. Molecular orbitals which belong to the $e$ representation for the $\mathrm{W}_{9} \mathrm{~N}$ surface molecule where nitrogen is bonded at a $5 \mathrm{CN}$ site.

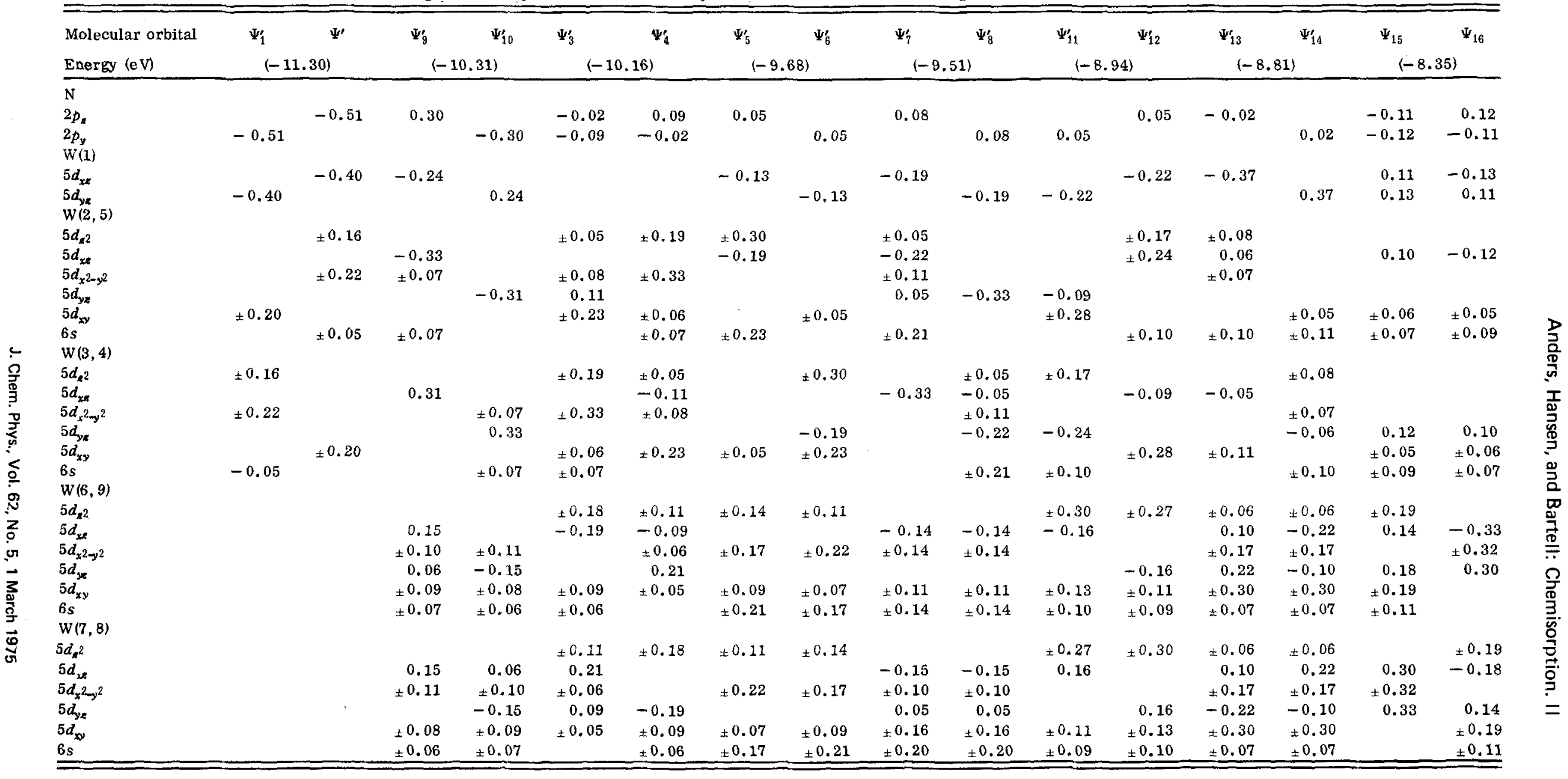


$5 \mathrm{CN}$ position, in which the nitrogen atom is well lowered into the surface, would lead to a double layer less negative out (and hence to a smaller increase in work function) than either the $2 \mathrm{CN}$ or $1 \mathrm{CN}$ position. The actual decrease in work function may stem from the fact that, using metal radius ( $1.37 \AA$ ) for tungsten and covalent radius $(0.70 \AA)$ for nitrogen, the top of the nitrogen atom proves to be $0.20 \AA$ below the tops of the surface tungsten atoms in the $5 \mathrm{CN}$ position.

* Prepared for the Energy Research and Development Administration, under contract number W-7405-eng-82.

${ }^{1}$ L. W. Anders, R. S. Hansen, and L. S. Bartell, J. Chem. Phys. 59, 5277 (1973).

${ }^{2}$ R. Hoffmann, J. Chem. Phys. 39, 1397 (1963).
${ }^{3}$ J. C. Slater, Phys. Rev. 36, 57 (1930).

${ }^{4}$ H. Basch, A. Viste, and H. B. Gray, Theor. Chim. Acta 3 , 458 (1965).

${ }^{5} \mathrm{~L}$. Pauling, The Nature of the Chemical Bond (Cornell U. P., Ithaca, NY, 1960), 3rd ed.

${ }^{6}$ T. A. Delchar and G. Ehrlich, J. Chem. Phys. 42, 2686 (1965).

${ }^{7}$ L. R. Clavenna and L. D. Schmidt, Surf. Sci. 22, 365 (1970).

${ }^{8}$ P. J. Estrup and J. Anderson, J. Chem. Phys. 46, 567 (1967).

${ }^{9}$ D. L. Adams and L. H. Germer, Surf. Sci. 26, 109 (1971).

${ }^{10}$ R. L. Park in Proc. Berkeley Int. Mater. Conf., 4th, University of California, Berkeley, June 1968 (1969), p. 28-1.

${ }^{11} \mathrm{~J}$. T. Yates, Jr., and T. E. Madey, Proc. Berkeley Int. Mater. Conf., 4th, University of California, Berkeley, June 1968 (1969), p. 19-1.

${ }^{12}$ R. S. Milliken, J. Chem. Phys, 23, 1833 (1955). 